\title{
Nutrition support in intensive care units in England: a snapshot of present practice
}

\author{
Mahtab N. Sharifi ${ }^{1}$, Anna Walton ${ }^{2}$, Gayatri Chakrabarty ${ }^{1}$, Tony Rahman ${ }^{3}$, Penny Neild ${ }^{1}$ \\ and Andrew Poullis ${ }^{1 *}$ \\ ${ }^{1}$ Department of Gastroenterology, St George's Hospital, Blackshaw Road, London SW17 OQT, UK \\ ${ }^{2}$ Department of Anaesthetics, St George's Hospital, Blackshaw Road, London, SW17 OQT, UK \\ ${ }^{3}$ Intensive Care Unit, St George's Hospital, Blackshaw Road, London SW17 OQT, UK
}

(Received 20 September 2010 - Revised 28 February 2011 - Accepted 28 February 2011 - First published online 31 May 2011)

\begin{abstract}
Nutrition support is an important part of care management in critically ill patients, not only to prevent and treat malnutrition but also it has a significant impact on recovery from illness and overall outcome. There is little information available about present nutritional support practice for patients in intensive care units (ICU) in the UK. This survey was designed to evaluate the present nutrition support practice in ICU and high dependency units (HDU) in England. Data were gathered by a $72 \mathrm{~h}$ phone survey from 245 ICU and HDU in $196 \mathrm{hospitals}$ in England. A questionnaire was completed over the telephone, including general information, nutrition support and teams involved in the nutrition management in the ICU. Of 1286 total patients in the ICU, 703 (54.6\%) were receiving nasogastric feeding, two (1.5\%) were receiving feeding via a percutaneous endoscopically placed gastrostomy tube and two (1.5\%) were receiving nasojejunal feeding. One hundred and forty-seven (11.4\%) patients were on parenteral feeding during the study period. A nutrition support team was not available in 158 ( $83.1 \%)$ ICU and there was no dietitian or specialist nutrition nurse to cover ICU in nine (4.7\%) hospitals. In conclusion, the present survey reported an increased trend in usage of enteral feeding in ICU in England, and a reduction in the use of parenteral nutrition compared with previous surveys. However, we are still far from integrating nutrition into care management in the ICU.
\end{abstract}

\section{Key words: Nutrition support: Intensive care units}

Malnutrition is common in hospitalised patients but is under-recognised and under-treated. It increases mortality and complications, and delays recovery from illness during and after hospital stay ${ }^{(1)}$. Various factors affect the outcome but malnutrition is considered an independent risk factor leading to higher complications and increased mortality, prolonged hospital stay and costs ${ }^{(2)}$. A recent study noted that patients over the age of 60 years with hip fracture who had protein-energy malnutrition had higher in-hospital mortality of $9.8 \%$ compared with $0 \%$ for patients without hip fracture and protein-energy malnutrition ${ }^{(3)}$.

Up to $40 \%$ of those admitted to hospitals in the UK are underweight $^{(4)}$. A study in New York reported $43 \%$ of admissions to ICU were malnourished ${ }^{(5)}$. The 'Malnutrition Universal Screening Tool' report showed that many will lose further weight while in hospital and up to $60 \%$ of hospital patients are clinically malnourished ${ }^{(6)}$. Malnutrition is also a considerable issue for surgical patients, particularly patients with malignancy. A study in Australia reviewed the nutrition status and peri-operative nutrition protocol in patients admitted for elective upper gastrointestinal or colorectal cancer surgery. They reported that poor nutritional status coupled with delayed and inadequate post-operative nutrition are associated with worse clinical outcomes ${ }^{(7)}$.

Nutrition support is an important part of care management in the critically ill, not only to prevent or treat malnutrition but also has a significant impact on recovery from illness and overall outcome. Enteral nutrition (EN) is nutrition provided through the gastrointestinal tract via a tube, catheter or stoma that delivers nutrients distal to the oral cavity ${ }^{(8)}$. Parenteral nutrition (PN) is the intravenous administration of nutrients via a central or peripheral vein.

Critical illness is a catabolic stress state with a systemic inflammatory response, which is associated with increased infection, multi-organ dysfunction, prolonged hospitalisation and mortality. Nutrition support is considered in an attempt to reduce the metabolic response to stress and to modulate the immune response. Stress response in a critically ill patient can be modulated with early EN and use of appropriate macro- and micronutrients. Early nutrition using the enteral

Abbreviations: EN, enteral nutrition; HDU, high dependency unit; ICU, intensive care unit; NG, nasogastric; NJ, nasojujenal; PEG, percutaneous endoscopically placed gastrostomy tube; PN, parenteral nutrition; TPN, total parenteral nutrition. 
route has been shown to reduce disease severity, diminish complications, decrease length of stay in the ICU and make a favourable impact on patient outcome.

There is little information available about the present nutritional support practice for patients in ICU in the UK. However, a nutrition survey reported that $43 \%$ of patients received nutritional support in $175 \mathrm{ICU}$ in England and Wales during a $24 \mathrm{~h}$ period $^{(9)}$.

This survey was designed to evaluate the present nutrition support practice in ICU and high dependency units (HDU) in England, and to identify areas for quality improvement initiatives.

\section{Method}

ICU ( $n$ 245) and HDU ( $n$ 196) hospitals in England were contacted by telephone. The telephonic survey was conducted by a medical doctor over a $72 \mathrm{~h}$ period during the last week of May 2006. The Directory of Critical Care Units published in 2004 was used to obtain phone numbers. These units were ICU, HDU or joint ICU/HDU admitting adult patients. Specialised paediatric ICU and coronary care units were not included in this survey.

A questionnaire was completed over the telephone from the sister-in-charge or nurse manager of the ICU. The first half of the questionnaire sought general information such as bed numbers, occupancy and type of patient. The second half of the survey consisted of questions about the type of nutrition support and teams involved in nutrition management. For the purpose of the study, nutritional support is defined as all forms of enteral tube feeding and all forms of parenteral feeding.

Ethics approval was not deemed necessary as this was an audit project.

No statistical analysis was required. All the values are expressed as absolute numbers or percentages.

\section{Results}

\section{General information}

Out of the total 245 ICU and HDU contacted, 190 (77.5\%) gave information and fifty-five $(22.4 \%)$ did not participate in the study (the most common reason cited being a lack of time to complete the telephone survey). A total of fifty-nine general ICU, eighty joint ICU/HDU, thirty-six HDU, nine cardiothoracic ICU, four neurosurgical ICU, one liver and one orthopaedic ICU participated in this survey. The total number of patients in the ICU or HDU was 1286 during the study period. The majority of the patients, 708 (55\%), were initially admitted to the ICU with surgical problems and 578 (45\%) patients with general medical problems (Table 1).

\section{Type of nutrition support}

Eight hundred and fifty-four (66.5\%) patients received EN or PN during the survey period. The majority received EN with 703 patients $(54.6 \%)$ received nasogastric (NG) feeding, two (1.5\%) received feeding via a percutaneous endoscopically placed gastrostomy tube (PEG) and two received (1.5\%) nasojejunal (NJ) feeding. One hundred and forty-seven (11.4\%) patients were on PN (Table 2).

\section{Nutrition support in intensive care units}

Of the 587 patients in a total of 74 ICU, 350 (59\%) received NG feeding, sixty-three (10.7\%) were on PN and one (0.1\%) on PEG feeding.

\section{Nutrition support in joint intensive care units/high dependency care units}

In eighty joint ICU/HDU, 297 (54\%) of patients were on NG feeding and seventy (12.7\%) were on PN. One (0.1\%) patient was reported on PEG feeding and one (0.1\%) on NJ feeding.

\section{Nutrition support in high dependency care units}

The lowest rates of EN and PN were reported in HDU. Of the 150 patients in HDU, fifty-six (37.3\%) were on NG feeding and fourteen $(9 \cdot 3 \%)$ were on PN. One patient (0.6\%) was reported on NJ feeding.

\section{Nutrition support team}

In the present study, a nutrition support team was defined as 'a multidisciplinary team including a doctor, a nurse, a pharmacist and a dietitian dealing specifically with nutrition

Table 1. Demographics of intensive care units (ICU) and high dependency units (HDU) in survey

(Numbers and percentage values)

\begin{tabular}{|c|c|c|c|c|c|c|c|c|}
\hline \multirow[b]{2}{*}{ Type of ICU } & \multirow{2}{*}{$\begin{array}{l}\text { Number } \\
\text { of units }\end{array}$} & \multirow{2}{*}{$\begin{array}{l}\text { Total number } \\
\text { of beds }\end{array}$} & \multirow{2}{*}{$\begin{array}{c}\text { Total number } \\
\text { of patients }\end{array}$} & \multirow[b]{2}{*}{ Occupancy (\%) } & \multicolumn{2}{|c|}{$\begin{array}{c}\text { Medical patients } \\
(\%)\end{array}$} & \multicolumn{2}{|c|}{$\begin{array}{c}\text { Surgical patients } \\
(\%)\end{array}$} \\
\hline & & & & & $n$ & $\%$ & $n$ & $\%$ \\
\hline Joint ICU/HDU & 80 & 669 & 549 & 82 & 260 & $47 \cdot 3$ & 289 & $52 \cdot 7$ \\
\hline HDU & 36 & 166 & 150 & $90 \cdot 3$ & 74 & $49 \cdot 3$ & 76 & $50 \cdot 7$ \\
\hline General ICU & 59 & 489 & 436 & $89 \cdot 1$ & 231 & $52 \cdot 9$ & 205 & $47 \cdot 1$ \\
\hline Cardiothoracic ICU & 9 & 113 & 92 & $81 \cdot 4$ & 0 & 0 & 92 & 100 \\
\hline Neurosurgical ICU & 4 & 37 & 34 & $91 \cdot 8$ & 0 & 0 & 34 & 100 \\
\hline Liver ICU & 1 & 15 & 13 & $86 \cdot 6$ & 13 & 100 & 0 & 0 \\
\hline Orthopaedic ICU & 1 & 12 & 12 & 100 & 0 & 0 & 12 & 100 \\
\hline Total & 190 & 1501 & 1286 & 85.6 & 578 & 44.9 & 708 & $55 \cdot 1$ \\
\hline
\end{tabular}


Table 2. Feeding routes used in different types of intensive care units (ICU) and high dependency units (HDU) (Numbers and percentage values)

\begin{tabular}{|c|c|c|c|c|c|c|c|c|c|}
\hline \multirow[b]{3}{*}{ Type of ICU } & \multirow[b]{3}{*}{ Number of units } & \multicolumn{8}{|c|}{ Patients receiving nutritional support } \\
\hline & & \multicolumn{2}{|c|}{$N G$} & \multicolumn{2}{|c|}{ PN } & \multicolumn{2}{|c|}{ PEG } & \multicolumn{2}{|c|}{ NJ } \\
\hline & & $n$ & $\%$ & $n$ & $\%$ & $n$ & $\%$ & $n$ & $\%$ \\
\hline Joined ICU/HDU & 80 & 297 & $54 \cdot 0$ & 70 & $12 \cdot 7$ & 1 & 0.1 & 1 & $0 \cdot 1$ \\
\hline HDU & 36 & 56 & $37 \cdot 3$ & 14 & 9.3 & 0 & 0 & 1 & 0.6 \\
\hline General ICU & 59 & 273 & $62 \cdot 6$ & 57 & $13 \cdot 0$ & 1 & 0.2 & 0 & 0 \\
\hline Cardiothoracic ICU & 9 & 41 & 44.5 & 3 & $3 \cdot 2$ & 0 & 0 & 0 & 0 \\
\hline Neurosurgical ICU & 4 & 25 & 73.5 & 0 & 0 & 0 & 0 & 0 & 0 \\
\hline Liver ICU & 1 & 10 & 76.9 & 1 & 7.6 & 0 & 0 & 0 & 0 \\
\hline Orthopaedic ICU & 1 & 1 & 8.3 & 2 & $16 \cdot 6$ & 0 & 0 & 0 & 0 \\
\hline Total & 190 & 703 & 54.6 & 147 & 11.4 & 2 & 1.5 & 2 & 1.5 \\
\hline
\end{tabular}

EN, enteral nutrition; PN, parenteral nutrition; NG, nasogastric; PEG, percutaneous endoscopically placed gastrostomy tube;

$\mathrm{NJ}$, nasojujenal.

issues on the ICU'. The survey showed that $158(83 \cdot 1 \%)$ ICU did not have a nutrition support team, and in nine (4.7\%) hospitals, there was no dietitian or specialist nutrition nurse to cover the ICU.

\section{Comparison with previous studies}

Table 3 compares the present study with the Hill et al. study ${ }^{(9)}$. Both studies looked at nutritional support in acutely ill patients. In the present study, $66 \%$ of the patients were on nutritional support, whereas in that of Hill et al. study, only $43 \%$ of the patients were on nutritional supplement. Also, there is more use of EN (82.8\%) compared with PN (17.2\%) in the present study. In the study of Hill et al. study, however, there was more use of PN (46\%) as opposed to EN (34\%). There was no use of the jejunostomy feeding tube in the present study group, but in Hill's study, seven patients received jejuostomy feeding.

\section{Discussion}

Nutrition support is a key area of management in critically ill patients, either to treat existing malnutrition or to prevent nutritional deficiencies. The present study showed that $66.5 \%$ patients in ICU in England were on one form of EN or PN support, which was a higher rate of nutrition support usage in the ICU than in the study of Hill et al. in 1995 (43\%).

The present study also showed that $54.9 \%$ of patients were on $\mathrm{EN}$ and $11.4 \%$ on $\mathrm{PN}$, while that of Hill et al. reported $34 \%$ of patients on only EN, $46 \%$ of patients on only PN and $16 \%$ were fed via more than one route. This demonstrates an increase in usage of EN and decrease in usage of PN in critically ill patients in ICU in England. However, it is important to note that, in the study of Hill et al., oral sip feed was also taken as a nutritional supplement, whereas in the present study that was not the case. This means that the difference may be far more than noted. Also in the present study group, none of the patients was on orogastric or jejunostomy feeding.

A number of clinical trials have indicated the benefits of providing nutrition support, particularly EN, to critically ill patients. A meta-analysis of twenty-seven studies comparing EN with PN in acutely ill patients reported a lower relative risk of infection with EN than with PN, regardless of nutritional status and year of study publication ${ }^{(10)}$. Heyland et $a l .{ }^{(11)}$ reviewed $\mathrm{PN} v$. EN in critically ill patients. The review suggested that when considering nutrition support in

Table 3. Comparison of present study with that of Hill et al. ${ }^{(9)}$ (Numbers and percentage values)

\begin{tabular}{|c|c|c|c|c|}
\hline & \multicolumn{2}{|c|}{ Present study } & \multicolumn{2}{|c|}{ Hill's study } \\
\hline & $n$ & $\%$ & $n$ & $\%$ \\
\hline Receiving nutritional support & 854 out of 1286 & $66 \cdot 5$ & 283 out of 659 & 43 \\
\hline \multicolumn{5}{|l|}{ Type of nutritional support in those receiving nutritional support } \\
\hline Only EN & $707 / 854$ & $82 \cdot 8$ & $97 / 283$ & $34^{\star}$ \\
\hline Only PN & $147 / 854$ & $17 \cdot 2$ & $131 / 283$ & $46^{*}$ \\
\hline \multicolumn{5}{|l|}{ Feeding route in } \\
\hline EN & 707 (only EN) & & $146(\operatorname{sips}+\mathrm{EN})$ & \\
\hline NG & 703 out of 1286 & $54 \cdot 6$ & 119 out of 146 & 81.55 \\
\hline PEG & 2 & & 1 & \\
\hline NJ & 2 & & 2 & \\
\hline Jejunostomy & 0 & & 7 & \\
\hline Orogastric tube & 0 & & 2 & \\
\hline
\end{tabular}

EN, enteral nutrition; PN, parenteral nutrition; NG, nasogastric; PEG, percutaneous endoscopically placed gastrostomy tube; NJ, nasojujenal. ${ }^{\star}$ Total less than $100 \%$ due to different definitions of nutrition support. 
critically ill patients, EN is used in preference to $\mathrm{PN}$ and has to be initiated within 24 to $48 \mathrm{~h}$ after admission to the ICU. They also advised not to use PN in combination with EN, particularly when initiating EN. Gramlich et al. ${ }^{(12)}$ reviewed thirteen studies showing that the use of EN in comparison with PN results in a significant decrease in infectious complication in critically ill patients but not with any difference in mortality rate. It also showed that EN may be less expensive.

Despite new ways of safe access to the gastrointestinal tract such as NJ tubes or PEG, few patients in the present study benefited from these routes. Only two patients received feeding via PEG and two patients via NJ. A meta-analysis reported that small bowel feeding may be associated with a reduction in gastro-oesophageal regurgitation, an increase in nutrient delivery and a shorter time to achieve the desired target nutrition in acutely ill patients ${ }^{(13)}$. It seems that these techniques are slowly being introduced to ICU settings in England.

Although EN is considered safe, EN-related bowel ischaemia has been reported with the use of surgical jejunostomy and NJ feeding tube especially in haemodynamically unstable patients including those on ionotropes ${ }^{(14,15)}$. However, a recent prospective study on haemodynamically unstable patients in medical ICU suggested that an early EN may be associated with reduced ICU and hospital mortality ${ }^{(16)}$. It is recommended that in the setting of haemodynamic compromise in which patients require haemodynamic support, including high-dose catecholamine agents, alone or in combination with a large volume of fluid resuscitation, EN should be withheld until the patient is fully resuscitated and/or stable ${ }^{(8)}$.

As might be expected, the lowest rate of artificial feeding routes was reported in HDU with $37 \cdot 3 \%$ of patients on NG, $9 \cdot 3 \%$ on $\mathrm{PN}$ and $0.6 \%$ on $\mathrm{NJ}$ feeding. This may reflect the fact that with improvement in the medical condition of patients and transfer from ICU to HDU or admission of patients with lower risk to HDU, the need to use artificial nutrition decreases.

This survey showed that only $16.9 \%$ of ICU had a nutrition team including a doctor, a dietitian, a nurse and a pharmacist. Of the ICU, $4.7 \%$ did not have either a dietitian or a specialised support nutrition nurse. Dietitians have an important role in nutrition assessment and calculating nutritional requirements of patients. Although the study by Woien \& Bjork ${ }^{(17)}$ reported an improvement in the delivery of EN to critically ill patients by nurses, using a nutritional support algorithm, this does not exclude the importance of the presence of a nutrition team in the ICU. The National Institute for Health and Clinical Excellence ${ }^{(18)}$ has recommended that, to tackle malnutrition in the National Health Service, all acute National Health Service Trust hospitals should recruit at least one specialist support nurse. This strategy can help delivery of nutrition support in ICU as well.

$\mathrm{PN}$ is an alternative or additional route of feeding when other routes are not successful or when it is not possible or unsafe to use EN. The aim of PN is to deliver nutrition closely related to the requirement safely. PN carries the risk of overfeeding, which is as deleterious as underfeeding. During acute illness, the aim should be to provide energy as close as possible to the measured energy expenditure to decrease negative energy balance ${ }^{(19)}$.

A study by Rubinson et al. ${ }^{(20)}$ of patients in ICU showed that those with low oral or enteral intake below 25\% of their requirements had a significant increase in the prevalence of bacteraemia. A prospective observational study showed that a negative energy balance was associated with an increase in all complications in a general ICU population ${ }^{(21)}$. However, studies have suggested a poor outcome from over-nutrition. Kirshman et al. ${ }^{(22)}$, in a prospective study showed that patients on $\mathrm{EN}$ or PN had better outcomes from $37.6 \mathrm{~kJ}$ (9 kcal) to $75.3 \mathrm{~kJ}(18 \mathrm{kcal}) / \mathrm{kg}$ per $\mathrm{d}$ than those receiving higher amounts.

Safety of PN can be ensured as follows: if by accurate assessment of the patient's nutritional needs, appropriate constitution PN is available, then there is safe intravenous access, proper catheter care and regular monitoring of electrolytes and anthropometric response. This could only be achieved through a coordinated nutrition team. A recent National Confidential Enquiry into Patient Outcome and Death report noted deficiencies in the assessment and monitoring of patients on $\mathrm{PN}$ in $54 \%$ of cases. Metabolic complications that occurred in 40 and $49 \%$ of these could have been avoided $^{(23)}$. Also, PN should only be considered where EN is noted to be inappropriate. The National Confidential Enquiry into Patient Outcome and Death report also noted that inadequate consideration was given to EN in one third of patients on $\mathrm{PN}^{(23)}$.

It is recommended that EN should be started early within the first $24-48 \mathrm{~h}$ following admission to an $\mathrm{ICU}^{(24)}$. Feeding started within this time frame compared with feeding started after $72 \mathrm{~h}$ is associated with reduced activation, and release of inflammatory cytokines, less gut permeability and reduced systemic endotoxaemia ${ }^{(11)}$. This meta-analysis also showed a reduction in infectious morbidity and mortality.

The aim of the present study was to assess nutrition use and support in ICU in the UK, however, with a broad usage of oral supplements in recent years; more research should be carried out to evaluate oral supplement usage in ICU in the UK.

In conclusion, this survey reports an increased trend in the usage of EN in ICU in England, and a reduction in the use of PN compared with previous surveys. However, we are still far from integrating nutrition into care management in ICU and the use of nutrition support teams and dedicated dietitians or specialist support nurses falls below National Institute for Health and Clinical Excellence guidelines.

\section{Acknowledgements}

The authors thank the staff, sister-in-charge or nurse managers of all ICU that participated in the present study for providing data and their important contributions. The authors had full access to all of the data in the study and take responsibility for the integrity of the data and the accuracy of the data analysis. The authors have no funding or support. The authors declare that they have no competing interests. A. P., P. N. and T. R. designed the study, interpreted the data analysis and revised the manuscript. M. N. S. designed the study, collected data, performed and interpreted the data analysis, 
and drafted the manuscript. G. C. performed data analysis and revised the manuscript. A. W. interpreted the data analysis and drafted the manuscript. P. N. revised the manuscript. All authors read and approved the final paper.

\section{References}

1. Elia M \& Stroud M (2004) Nutrition in acute care. Clin Med $\mathbf{4}$, 405-407.

2. Correia MI \& Waitzberg DL (2003) The impact of malnutrition on morbidity, mortality, length of hospital stay and costs evaluated through a multivariate model analysis. Clin Nutr 22, 235-239.

3. O'Daly BJ, Walsh JC, Quinlan JF, et al. (2010) Serum albumin and total lymphocyte count as predictors of outcome in hip fractures. Clin Nutr. 29, 89-93.

4. Elia M \& Stratton RJ (2000) How much under-nutrition is there in British hospitals? Br J Nutr 84, 257-259.

5. Giner M, Laviano A, Meguid MM, et al. (1996) In 1995 a correlation between malnutrition and poor outcome in critically ill patients still exists. Nutrition 12, 23-29.

6. Malnutrition Advisory Group, a standing committee of British Association for Parenteral and Enteral Nutrition (2003) Nutritional screening for adults - a multidisciplinary responsibility. Press release, MUST Launch London, Nov 11. http://bapen.org.uk/res_press_rel12.html

7. Garth AK, Newsome CM, Simmance N, et al. (2010) Nutritional status, nutrition practices and post-operative complications in patients with gastrointestinal cancer. J Hum Nutr Diet 23, 393-401.

8. Teitelbaum D, Guenter P, Howell WH, et al. (2005) American Society for Parenteral and Enteral Nutrition Board of Directors and Standards Committee: Definition of terms, style, and conventions used in A.S.P.E.N. guidelines and standards. Nutr Clin Pract 20, 281-285.

9. Hill SA, Nielsen MS \& Lennard-Jones JE (1995) Nutritional support in intensive care units in England and Wales: a survey. Eur J Clin Nutr 49, 371-378.

10. Braunschweig CL, Levy P, Sheean PM, et al. (2001) Enteral compared with parenteral nutrition: a meta-analysis. $\mathrm{Am} \mathrm{J}$ Clin Nutr 74, 534-542.

11. Heyland DK, Dhaliwal R, Drover JW, et al. (2003) Canadian Critical Care Clinical Practice Guidelines Committee. Canadian clinical practice guidelines for nutrition support in mechanically ventilated,critically ill adult patients. JPEN J Parenter Enteral Nutr 27, 355-373.
12. Gramlich L, Kichian K, Pinilla J, et al. (2004) Does enteral nutrition compared to parentral nutrition result in better outcome in critically ill adult patients? A systemic review of literature. Nutrition 20, 843-848.

13. Heyland DK, Drover JW, Dhaliwal R, et al. (2004) Optimizing the benefits and minimizing the risks of enteral nutrition in the critically ill: role of small bowel feeding. JPEN Parenter Enteral Nutr 26, Suppl. 6, S51-S57.

14. Melis M, Fichera A \& Ferguson MK (2006) Bowel necrosis associated with early jejunal tube feeding: a complication of postoperative enteral nutrition. Arch Surg 141, 701-704.

15. Zaloga GP, Roberts PR \& Marik P (2003) Feeding the hemodynamically unstable patient: a critical evaluation of the evidence. Nutr Clin Pract 18, 285-293.

16. Khalid I, Doshi P \& DiGiovine B (2010) Early enteral nutrition and outcomes of critically ill patients treated with vasopressors and mechanical ventilation. Am J Crit Care 19, 261-268.

17. Woien H \& Bjork IT (2006) Nutrition of critically ill patient and effect of implementing a nutritional support algorithm in ICU. J Clin Nurs 15, 168-177.

18. National Institute for Clinical Excellence (NICE) (2006) CG32 Nutrition Support in Adult: Full Guidance, pp. 56-61. London: NICE.

19. Cano NJM, Aparicio M, Brunori G, et al. (2009) ESPN guidelines for adult parenteral nutrition. Clin Nutr 28, 359-479.

20. Rubinson L, Diette GB, Song XS, et al. (2004) Low calorie intake is associated with noscomial bloodstream infections in patients in the medical intensive care unit. Crit Care Med 32, 350-357.

21. Dvir D, Cohen J \& Singer P (2006) Computerized energy balance and complication in critically ill patients: an observational study. Clin Nutr 25, 37-44.

22. Kirshman JA, Parce PB, Martinez A, et al. (2003) Caloric intake in medical ICU patients. Consistency of care with guidelines and relationship to clinical outcomes. Chest $\mathbf{1 2 4}$, $297-305$.

23. National Confidential Enquiry into Patients Outcome and Death (NCEPOD) report (2010) Parenteral nutrition: a mixed bag 2010, Parenteral Nutrition toolkit. http://ncepod. org.uk/2010pn.htm

24. McClave SA, Martindale RG, Vanek VW, et al. (2009) Guidelines for the Provision and Assessment of Nutrition Support Therapy in the Adult Critically Ill Patient: Society of Critical Care Medicine (SCCM) and American Society for Parenteral and Enteral Nutrition (ASPEN). JPEN J Parenter Enteral Nutr 33, 277-316. 